\title{
Las escuelas unidocentes en Costa Rica: fortalezas y limitaciones
}

\section{Single-teacher schools in Costa Rica: strenghts and limitations}

\author{
Lupita Chaves Salas ${ }^{1}$ \\ Universidad de Costa Rica, Escuela de Formación Docente \\ San José, Costa Rica \\ lupitachaves@gmail.com
}

Jacqueline García Fallas ${ }^{2}$

Universidad de Costa Rica, Escuela de Filosofía

San José, Costa Rica

jgarciafallas@gmail.com

Recibido: 01 mayo 2012 Aceptado: 28 febrero 2013 Corregido: 14 mayo 2013

\begin{abstract}
Resumen: Este artículo presenta los resultados de la investigación "Las escuelas unidocentes de Costa Rica: fortalezas y limitaciones”. Se realiza una investigación sustentada metodológicamente en un enfoque mixto. Consta de tres partes: en la primera se identifican algunos aspectos generales de esos centros educativos con base en datos del Departamento de Estadistica del Ministerio de Educación Pública (MEP), tales como: categoría profesional del personal docente, cobertura, ubicación, porcentajes de aprobación, exclusión y repitencia. En la segunda parte se selecciona una muestra representativa de unidocentes de todo el país y se indaga sobre: gestión y organización administrativa, infraestructura, material didáctico y tecnológico, planeamiento, práctica pedagógica, relación con familias y comunidad, entre otros. En un tercer momento, se realiza un estudio etnográfico en cuatro instituciones unidocentes consideradas exitosas por el Ministerio de Educación Pública, con el fin de observar e indagar cómo se desarrolla el currículo en esos centros educativos. El estudio concluye que aunque se han realizado algunos esfuerzos para mejorar la calidad de estos centros educativos por parte del MEP, las escuelas unidocentes no han sido prioridad para el Estado.
\end{abstract}

Palabras claves: Escuela unidocente, escuela multigrado, prácticas pedagógicas, administración educativa, Ministerio de Educación Pública, Costa Rica.

Doctora en Educación. Magister en Educación de Adultos. Bachiller y Licenciada en Educación Preescolar. Actualmente es Decana de Facultad de Educación de la Universidad de Costa Rica y directora del Programa de Posgrado Académico en Educación. Es investigadora del Instituto de Investigación en Educación de la UCR y fue su directora del 2001 al 2008. Docente de la carrera de Bachilllerato y Licenciatura en Educación Preescolar de la Escuela de Formación Docente. Directora de tesis de grado y posgrado. Participa como ponente en congresos nacionales e internacionales. Es miembro del Consejo Editorial de la Revista en español de la International Reading Association y de la Revista de Educación de la Universidad Central de Venezuela. Es autora de libros, capítulos de libros y numerosos artículos en revistas nacionales y de otros países sobre una diversidad de temas: educación preescolar, procesos iniciales de lectoescritura, investigación-acción en el aula, formación docente, escuelas unidocentes, docentes destacados, evaluación en secundaria, entre otros. Coordina proyectos de extensión dirigidos a docentes en zonas urbanas y rurales.

2 Doctora en Educación de la Universidad de Costa Rica. Labora como investigadora en el Instituto de Investigación en Educación y como docente en la Escuela de Filosofía de la Universidad de Costa Rica. Ha participado como ponente en congresos nacionales e internacionales. Entre sus publicaciones recientes se destacan: "Uso de un portafolio virtual para incorporar la dimensión ambiental: una experiencia de investigación-acción en el aula”. En Revista Actualidades Investigativas en Educación, Vol. 11, número especial, 2011. Construcción de una comunidad virtual de aprendizaje ambiental (CVAA): espacio interactivo para ambientalizar el quehacer universitario estatal de Costa Rica En Revista Actualidades Investigativas en Educación, vol. 11, número especial, 2011. Educación ambiental: una estrategia para ambientalizar el currículo universitario. En Biocenosis, Revista de Educación Ambiental, Centro de Educación Ambiental, Universidad Estatal a Distancia, Costa Rica, vol. 26 (1-2), 2012. Percepción sobre la dimensión ambiental en el ámbito personal y laboral: aplicación y valoración de un pretest. En Biocenosis, Revista de Educación Ambiental, Centro de Educación Ambiental, Universidad Estatal a Distancia, Costa Rica, vol. 26 (1-2), 2012, entre otras. 


\begin{abstract}
This article presents the results of the research work "Las escuelas unidocentes de Costa Rica: fortalezas y limitaciones" (Single-teacher schools in Costa Rica: strenghts and limitations). Research's methodology is based on mixed methods. It has three parts; in the first one, general aspects of those teaching schools are identified using data from the Statistics Department of the Ministry of Education (MEP), such as teaching staff's professional profile, coverage, location, and rates for course completion, exclusion and failure in the second part, a meaningful sample of singleteacher subjects from all over the country is selected, and data on management and organization, infrastructure, teaching and technological material, planning, pedagogical practice, community and family relationships is collected, among other things. In the third instance, an ethnographic study is carried out in four single-teacher institutions graded as successful by the Ministry of Public Education, with the goal of observing and researching how the curriculum is being developed in these teaching schools.

The study concludes by saying that, despite some efforts to improve those educational schools have been made by the Ministry of Education, the State has not considered single-teacher schools a priority.
\end{abstract}

Keywords: single-teacher school, multiple-course school, pedagogical practices, education management, Ministry of Public Education, Costa Rica.

\title{
Introducción
}

Las instituciones unidocentes se han conocido históricamente como centros educativos donde se ofrece el I y II ciclo de la Educación General Básica (EGB) a niñas y niños que son atendidos únicamente por una maestra o un maestro, por lo general se ubican en zonas rurales donde la población estudiantil es escasa, el cual es un criterio importante para su creación. Estas instituciones tienen sus diferencias con respecto a otras del sistema público, pues en una misma aula se ubican a todos los estudiantes desde primero hasta sexto, con edades comprendidas entre los 6 y los 14 años, por lo que la organización administrativa, curricular y didáctica es distinta a la que tienen los centros que agrupan al alumnado por niveles. En estos centros, la matrícula no es mayor de treinta estudiantes y el MEP recomienda un horario de 7:00 a.m. a 1:15 p.m.

En 1994 en el marco de la política educativa "Hacia el Siglo XXI" y como respuesta al bajo rendimiento escolar de la población estudiantil de las escuelas unidocentes en relación con el estudiantado de I y II ciclo que asiste a otros centros educativos, se incorpora un segundo educador o educadora a esas escuelas que tuvieran más de cuarenta estudiantes (MEP, 1997). Asimismo, en el año 2000 se integra el nivel de educación preescolar en 28 escuelas unidocentes mediante la Estrategia de Grupos Heterogéneos (Niñas y niños de 4 años 3 meses a 6 años 3 meses en una misma sección), como plan piloto y de investigación. Esta experiencia fue exitosa, por lo que se amplía su cobertura, y para el año 2007 había 996 escuelas unidocentes y de dirección 1 con educación preescolar (MEP, 1997).

Por lo anteriormente citado, a las escuelas unidocentes, en la actualidad, también se les conoce como escuelas multigrado. Si bien es cierto que las escuelas unidocentes o multigrado vienen a ampliar el acceso a la educación primaria en zonas rurales de nuestro país, también se han evidenciado problemas en cuanto a la calidad de educación que brindan. En un 
documento del Ministerio de Educación (1997, p. 1) se indica:

Los estudios y diagnósticos sobre rendimiento académico y calidad de la educación en general, evidencian que precisamente son los estudiantes de estos centros educativos (escuelas unidocentes) los que se encuentran en mayor desventaja con respecto al resto del estudiantado nacional.

Otras investigaciones realizadas en la Universidad de Costa Rica (Sanabria, 1981 y Abarca, Gómez y Peralta, 1987), en la década de los ochenta, encuentran que los problemas que sufrían las escuelas unidocentes en ese momento eran los siguientes:

- Siguen los mismos planes y programas de estudio de las escuelas pluridocentes urbanas y rurales.

- Currículo poco flexible.

- Personal docente con poca formación, muchos son aspirantes.

- Deficientes programas de educación continua para el personal docente que atiende estas instituciones.

- Infraestructura inadecuada.

- Pocos recursos didácticos y equipo.

- No hay incentivos para el personal docente.

- La atención de niñas y niños de los seis niveles de la educación primaria simultáneamente, sin contar con la formación y ni asesoría necesaria.

- Problemas en cuanto al acceso, alimentación y vivienda para el personal docente.

La inequidad del sistema educativo se evidencia también en estudios realizados por el Banco Mundial (2003) y por el Programa de Estado de la Nación (2002) sobre la educación rural en Costa Rica.

La investigación financiada por el Banco Mundial muestra que las menores tasas de asistencia a la educación se concentran sobre todo en las zonas rural y rural dispersa, en esta última la no asistencia es más del doble que en la zona urbana. Entre las conclusiones se indica que la combinación zona rural-pobreza es lo que excluye a más personas de la educación.

El Informe del Proyecto del Estado de la Nación del 2002 señala que las escuelas unidocentes presentan mayores porcentajes de deserción, reprobación y repitencia en comparación con el promedio nacional de la educación primaria y estos datos se mantienen constantes al compararlos con años anteriores.

Asimismo, las pruebas internacionales elaboradas por el Laboratorio Latinoamericano de Evaluación de la Calidad y la Educación (LLECE), que se aplicaron en diferentes países de la región en el año 2006, muestran que la población estudiantil de Costa Rica de tercer y sexto grado de zonas rurales obtiene menores puntajes en matemática y lectura que los de zonas urbanas (Programa Regional Educativo para América Latina y el Caribe, 2008). 
Estos datos muestran las diferencias que se presentan en el sistema educativo costarricense, lo que evidencia que la escuela está contribuyendo a la reproducción de las desigualdades sociales (Apple, 1995; Giroux, 1995; McLaren, 1997) al ofrecer a la población indígena, rural o marginal una educación poco pertinente y relevante de acuerdo con las demandas actuales. Esta evidencia se relaciona también con la pobreza, los programas de estudio, la formación profesional del personal docente, la falta de material didáctico y tecnológico, entre otros aspectos.

Con base en lo citado, este estudio se planteó los siguientes objetivos:

\section{Objetivos}

\section{Objetivo general}

Analizar la modalidad de escuelas unidocentes del Ministerio de Educación Pública para identificar fortalezas y limitaciones en cuanto al servicio educativo que ofrece.

\section{Objetivos específicos}

1. Identificar la cobertura y la ubicación de las escuelas unidocentes en Costa Rica.

2. Identificar los porcentajes de aprobación, exclusión y repitencia de la población estudiantil de las escuelas unidocentes del 2000 al 2006.

3. Identificar las características sociodemográficas del personal docente que atiende a las escuelas unidocentes.

4. Analizar los programas de estudio que recomienda el Ministerio de Educación Pública para las escuelas unidocentes a la luz de las nuevas tendencias pedagógicas.

5. Identificar las actividades de educación continua a las que asiste el personal docente de las escuelas unidocentes.

6. Analizar la gestión y organización administrativa de las escuelas unidocentes exitosas.

7. Analizar la práctica pedagógica del personal docente de las escuelas unidocentes exitosas en cuanto a: planeamiento, labor de aula, organización del ambiente y del tiempo, relación estudiante-docente, material didáctico y trabajo con familias $\mathrm{y}$ comunidad.

\section{Características de las escuelas unidocentes}

La escuela unidocente en Costa Rica es aquella institución de I y II ciclos que alberga una población escolar de treinta alumnos o menos, los cuales son atendidos por un solo maestro o maestra, en una o dos jornadas. Si labora con una sola jornada, es decir con un grupo menor de veinticinco estudiantes, el docente trabaja con los seis niveles escolares de 7:00 a.m. a 1:15 p.m. y de 1:15 a 2:40 p.m. se dedica a las tareas de planificación didáctica. Cuando trabajan con dos 
jornadas, atienden tres niveles por la mañana y tres niveles por la tarde. La primera de 7:00 a.m. a 10:10 a.m. (3 días), de 7:00 a.m. a 10:50 a.m. (dos días) y la segunda de 10:50 a.m. a 2:00 p.m. y dos días de 11:30 a.m. a 3:20 p.m.

Las escuelas unidocentes constituyen una alternativa que permite a los niños y las niñas de comunidades rurales dispersas acceder a la educación, quienes de otra manera no tendrían la oportunidad de recibir la educación primaria completa, pues sus hogares están muy alejados de los centros de población. Estas escuelas, en el año 2007, representaban el $49.63 \%$ del total de estos centros en nuestro país.

El personal docente, al tener que atender a seis o tres niveles a la vez, asume un papel muy diferente al que tienen los profesionales en educación de otras escuelas, ya que debe planificar el trabajo diario de tal forma que pueda estimular, orientar y mediar los aprendizajes de una forma no directiva, puesto que no puede atender directamente a todos los niveles a la vez. Esta particularidad permite además que el docente delegue mayores responsabilidades en los educandos y estos asuman el verdadero papel que les corresponde, el de "actores principales del proceso educativo". Asimismo, el docente debe asumir la gestión administrativa.

El personal docente debe favorecer la conformación de verdaderas comunidades de aprendizaje, en las cuales todos aprenden, todos enseñan y todos tienen algo que aportar para lograr los grandes objetivos de la educación primaria. También se ven en la obligación de desarrollar la creatividad. Al no contar con materiales suficientes, se buscan diferentes alternativas para solventar las necesidades que demandan sus funciones en el campo técnico pedagógico, administrativo y psicosocial, obteniendo el mejor provecho de los pocos recursos con que cuenta la institución y los que ofrece la comunidad.

El alumnado en estas escuelas asume un papel diferente, ya que la dinámica escolar requiere de niños y niñas autónomos, solidarios, que pueden seguir indicaciones, que investigan, analizan, preguntan, conocen donde pueden encontrar la información y la ayuda que requieren, la cual no se circunscribe únicamente al docente. Además, deben trabajar adecuadamente en forma individual o en grupos.

Una particularidad del estudiantado de estas escuelas es que se dedican a actividades productivas ligadas con la agricultura, la ganadería, la pesca, entre otros, en las cuales participan todos los miembros de la familia, por lo que las niñas y los niños no solo estudian, sino que ayudan a sus padres y madres en las tareas del hogar y en el trabajo.

Una de las grandes ventajas que mantiene aún la escuela unidocente es la de tener una comunidad educativa claramente definida, lo que permite al personal docente plantear estrategias para unir esfuerzos en pro del desarrollo institucional y comunal.

Las escuelas unidocentes, como se describió anteriormente, poseen características muy propias que hacen que estas instituciones sean de suma importancia para todos los actores que intervienen en el proceso educativo y para el país, ya que ofrecen la oportunidad a miles de niños y de niñas de zonas rurales dispersas de ampliar la visión del mundo que los rodea, 
adquirir y fortalecer habilidades y destrezas que les permitan una mejor incorporación económica y social a sus comunidades, y puedan desarrollarse plenamente a nivel afectivo, psicomotor y cognoscitivo.

\section{Metodología}

Para analizar las fortalezas y las limitaciones de las escuelas unidocentes en Costa Rica el estudio se organiza en tres partes.

En la primera parte se lleva a cabo una investigación descriptiva para conocer, a nivel general, algunas de las características de las escuelas unidocentes: la cobertura y la ubicación, los porcentajes de aprobación, exclusión y repitencia de la población estudiantil en los últimos seis años, las características profesionales del personal docente. Asimismo, se analizan los programas de estudio que recomienda el Ministerio de Educación Pública y se describen las actividades de educación continua que recibe el personal docente de esas instituciones.

En la segunda parte se selecciona una muestra representativa de unidocentes de todo el país y se distribuyó un cuestionario en el que se indagó sobre: gestión y organización administrativa, infraestructura, material didáctico y tecnológico, planeamiento, práctica pedagógica, relación con familias y comunidad, entre otros. Para obtener esta información se elaboró un cuestionario con base en los objetivos de esta investigación.

En la tercera parte se realiza un estudio etnográfico en cuatro escuelas unidocentes consideradas exitosas por parte de autoridades del Ministerio de Educación Pública, con el fin de vivenciar las experiencias cotidianas del aula con el personal docente, la población estudiantil y las familias. Para la selección de estas escuelas se recurrió, en un primer momento, a los datos del Departamento de Estadística del MEP, con el propósito de conocer los porcentajes de rendimiento académico de todas las escuelas unidocentes del país, analizar los resultados y escoger las escuelas con mejores porcentajes; sin embargo, esta indagación no dio los resultados esperados debido a que el comportamiento es similar en los diferentes centros educativos. Asimismo, se considera importante la opinión de autoridades (Director (a) Regional y Asesor Supervisor) de las Direcciones Regionales Educativas, a los cuales se les solicita información en torno a las escuelas a su cargo, para conocer su criterio sobre las que tienen mejor desempeño. Un aspecto que se consideró fue la participación del personal docente en proyectos que impulsa el MEP para el mejoramiento de la calidad de la educación, y el compromiso manifestado por el personal docente con su labor profesional. Finalmente, la selección de las regiones donde se haría el trabajo de campo se hizo pensando en que representara a diferentes zonas del país, además se tomó en cuenta la disponibilidad del personal docente y de las autoridades regionales; también en esta decisión fue importante la disponibilidad presupuestaria y la conveniencia de las investigadoras. 


\section{Etapas de la investigación}

I Etapa: Elaboración histórica, teórica y estadística. Revisión de bibliografía y de estadísticas del Ministerio de Educación Pública (MEP). Elaboración de una reseña histórica, de un marco de referencia teórico y de tablas sobre categoría profesional del personal docente, cobertura, ubicación de las escuelas unidocentes y del rendimiento académico del estudiantado: porcentajes de aprobación, reprobación, exclusión y repitencia.

II Etapa: Elaboración y validación de instrumentos. A partir de la revisión bibliográfica y consulta a asesores del Ministerio de Educación Pública se construye un cuestionario para que completen las educadoras y los educadores seleccionados en la muestra. La validación de este instrumento se realizó mediante juicio de expertos. El instrumento cuenta con 86 preguntas.

III Etapa: Selección de la muestra y recolección de la información. La población de esta investigación la constituyen las escuelas multigrado del país distribuidas en las 20 Regiones Educativas del Ministerio de Educación Pública en el año 2007.

Para calcular las personas que participaron en el estudio se utilizó un muestreo irrestricto aleatorio (mia) con selección aleatoria proporcional al tamaño de la región. Esto significa que de acuerdo con la cantidad de centros educativos dentro de cada región, así se seleccionaron en la muestra, de tal forma que se tuvo representación de todas las regiones. Por ejemplo, en la región Aguirre hay 62 centros educativos, esto representa un $3 \%$ de los centros unidocentes del país. Entonces, el 3\% de la muestra debe contener centros unidocentes de Aguirre, lo que significa 14 centros educativos (Muestra elaborada por Juan Pablo Solís, de la Escuela de Estadística, con los datos proporcionados por el Ministerio de Educación). La selección de las escuelas dentro de las regiones se realizó al azar. Para 1854 centros educativos multigrado, la muestra representativa sería de 400 unidocentes; como mínimo debían completar el instrumento 320 personas y lo hicieron un total de 335 .

La distribución de la muestra seleccionada se muestra a continuación: 
Tabla 1

Distribución de la muestra

\begin{tabular}{|c|c|c|c|}
\hline Región & Escuelas en la población & Porcentaje de escuelas & Escuelas en la muestra \\
\hline Aguirre & 33 & 2 & 7 \\
\hline Alajuela & 69 & 4 & 15 \\
\hline Cañas & 69 & 4 & 15 \\
\hline Cartago & 56 & 3 & 12 \\
\hline Coto & 248 & 13 & 55 \\
\hline Desamparados & 48 & 3 & 11 \\
\hline Guápiles & 68 & 4 & 15 \\
\hline Heredia & 63 & 3 & 14 \\
\hline Liberia & 39 & 2 & 9 \\
\hline Limón & 109 & 6 & 24 \\
\hline Nicoya & 127 & 7 & 28 \\
\hline Pérez Zeledón & 224 & 12 & 50 \\
\hline Puntarenas & 122 & 7 & 27 \\
\hline Puriscal & 78 & 4 & 17 \\
\hline San Caros & 182 & 10 & 40 \\
\hline San José & 40 & 2 & 9 \\
\hline San Ramón & 40 & 2 & 9 \\
\hline Santa Cruz & 45 & 2 & 10 \\
\hline Turrialba & 117 & 6 & 26 \\
\hline Upala & 77 & 4 & 17 \\
\hline Total & 1854 & 100 & 400 \\
\hline
\end{tabular}

Para distribuir los cuestionarios, estos se agruparon por región educativa y por circuito de acuerdo con la muestra. Además, se le adjuntó dos cartas: una dirigida al supervisor y la otra al personal docente junto con el cuestionario. En las cartas se indicaba el objetivo y la importancia del estudio, y la forma de hacer llegar el instrumento al Instituto de Investigación en Educación de la UCR, además de las listas de las escuelas seleccionadas. Algunos de los sobres con toda esta información los repartió la directora del programa Nacional de Escuelas Unidocentes en una actividad organizada por el Departamento de I y II ciclo del Ministerio de Educación, dirigida a maestras y maestros de Escuelas Unidocentes de todo el país celebrada en el Hotel Irazú, los días 4, 5 y 6 de junio del 2008. La gran 
mayoría de los sobres se dejaron personalmente en las Direcciones Regionales y otros se enviaron por correo. Se mantuvo contacto telefónico con las Direcciones Regionales y con las supervisaras y supervisores de cada uno de los circuitos seleccionados en la muestra, para la aplicación y envío de los instrumentos. Se decidió que los cuestionarios se completaran en reuniones que convocaba cada circuito.

Para recolectar los cuestionarios se ofrecieron las siguientes opciones: enviarlos en sobre cerrado al Departamento de I y II ciclo del Ministerio de Educación Pública, dirigido a la M.Sc. Rocío Alvarado, al fax 22074679, o acceder al instrumento en la página web www.inie.ucr.ac.cr, completar y enviar. La mayoría llegaron por correo y en algunos casos las investigadoras los recogieron personalmente. Conforme llegaban los instrumentos, se marcaban en la lista las escuelas que lo remitían, de esta forma se le dio seguimiento a los centros educativos que tardaron en enviarlos. Este proceso requirió de seguimiento semanal de parte de equipo de las investigadoras mediante llamadas telefónicas y visitas al personal docente y al asesor-supervisor de las Direcciones Regionales. En un periodo de seis meses se recolectaron los instrumentos, de agosto del 2008 a febrero del 2009.

El proceso de recolección de los cuestionarios fue un proceso difícil. En algunas regiones se enviaron hasta tres veces porque se perdían y no daban razón de ellos. Además el seguimiento implicó un sinnúmero de contactos telefónicos con directoras regionales, supervisoras y educadoras. Otra dificultad que se presentó es que en el año 2008 se crearon dos regiones más, a las que se le envió posteriormente los cuestionarios.

Además del personal docente que llenó los instrumentos, se le hizo entrevista a tres informantes clave: a la M. Sc. Alejandrina Mata Segreda, viceministra académica del Ministerio de Educación Pública; a la M.Sc. Rocío Alvarado Cruz, la encargada del Programa Nacional de Escuelas Unidocentes, y a la Dra. Zaida Molina, exfuncionaria del MEP que formó parte de la comisión que elaboró el Programa de Estudio para Escuelas Unidocentes, en la década de los ochenta.

También participaron en la investigación las cuatro unidocentes a las cuales se les observó su práctica pedagógica, y cincuenta y seis unidocentes de Tilarán, Limón, Palmar Norte y Pacayas, los cuales validaron la información recolectada en este estudio.

IV Etapa. Negociación de entrada: Se realiza la negociación de entrada a las cuatro instituciones unidocentes seleccionadas, para ello se contactó en primer término a la directora de la escuela y se le presentó el proyecto con el propósito de conocer su disposición para participar en el estudio. Posteriormente se solicita el permiso a las autoridades correspondientes de las Direcciones Regionales y cada docente firma el consentimiento informado.

V Etapa. Inmersión al salón de clase: Cada escuela se visitó durante dos semanas con el fin de observar la jornada completa de trabajo, la organización del ambiente y del tiempo, las relaciones entre las personas que comparten el espacio escolar, la labor pedagógica, el material didáctico y su uso. Asimismo, se hicieron entrevistas al personal docente, a la población estudiantil y a las familias. A todas las personas participantes en el estudio se les 
explicó el proyecto y firmaron el consentimiento informado. La observación del trabajo de aula fue realizada por las mismas investigadoras, de manera individual; una de ellas lo realizaba durante una semana y la otra, la siguiente semana, con el fin de contrastar la información obtenida. De la siguiente manera:

\author{
Escuela A \\ Semana Investigadora \\ 16 al 20 de febrero del 2009 Jacqueline García \\ 23 al 27 de febrero del 2009 Lupita Chaves \\ Escuela B \\ 23 al 27 de febrero del 2009 Jacqueline García \\ 2 al 6 de marzo del 2009 Lupita Chaves \\ Escuela C \\ 25 al 29 de mayo del 2009 Jacqueline García \\ 1 al 5 de junio del 2009 Lupita Chaves \\ Escuela D \\ 3 al 7 de agosto del 2009 Jacqueline García \\ 10 al 14 de agosto del 2009 Lupita Chaves
}

Para recolectar los datos se utilizó el diario de campo, donde se anotó lo que acontecía en el aula, la relación estudiante-estudiante, estudiante-docente, las prácticas pedagógicas, el clima afectivo del aula, entre otras. Estas notas se transcribían una vez finalizada la observación y se le agregaban notas teóricas y las percepciones de las investigadoras sobre lo que habían observado. Se utilizaron las siguientes técnicas e instrumentos: fotografía y vídeo, cuestionario dirigido a la unidocente, guías de entrevista a las familias y a la población estudiantil, guías de observación a la comunidad, a la institución y al aula.

Además se realizaron entrevistas no estructuradas a informantes claves.

VI Etapa. Análisis de la información: El análisis de la información cuantitativa se hizo interpretando las frecuencias absolutas y relativas de los datos obtenidos en el Departamento de Estadística del Ministerio de Educación Pública y de las repuestas dadas por los educadores y las educadoras en los cuestionarios. Para estos últimos se utilizó el programa SPSS y se contó con el apoyo de la Unidad de Servicios Estadísticos de la Escuela de Estadística de la Universidad de Costa Rica. Los datos cualitativos se analizaron triangulando la siguiente información: entrevistas a estudiantes, familias y cuestionario a docentes con observaciones a la labor de aula, documentos de la maestra, trabajo de la población estudiantil y con la teoría.

Los datos cuantitativos y cualitativos se presentan en forma integrada para ofrecer una visión completa del objeto estudiado.

VII Etapa. Validación de resultados: Una vez analizados los datos cuantitativos y cualitativos se formuló una lista de fortalezas y limitaciones de las escuelas unidocentes, 
con el propósito de validar estos datos con docentes que laboran en esta modalidad y que pertenecen a los circuitos educativos de las maestras que se les observó su práctica pedagógica. En Pacayas únicamente existe solo una escuela unidocente, como se evidencia en la tabla 2 :

Tabla 2

Unidocentes que validaron información

\begin{tabular}{lc}
\hline Lugar & No de docentes $^{\circ}$ \\
Tilarán & 22 \\
Palmar Norte & 18 \\
Limón & 15 \\
Pacayas & 1 \\
\hline Total & $\mathbf{5 6}$ \\
\hline
\end{tabular}

VIII Etapa, Preparar el informe final: Elaboración y presentación de informe final.

\section{Principales hallazgos}

Seguidamente se presentan los hallazgos más relevantes del estudio, de acuerdo con los objetivos de la investigación. Esta información se organiza en dos apartados: el primero recoge las generalidades de las escuelas unidocentes y el segundo aborda las fortalezas y las limitaciones de las escuelas unidocentes a partir del análisis cuantitativo y cualitativo (Chaves, García y Alvarado, 2010).

\section{Generalidades de las escuelas unidocentes}

\section{Cobertura y la ubicación de las escuelas unidocentes en Costa Rica}

Las escuelas unidocentes se ubican en todas las regiones del país. Tienen una mayor presencia en zona rural (91\%). Las regiones que cuentan con un mayor número de instituciones de este tipo son: Coto, Pérez Zeledón y San Carlos (Chaves, García y Alvarado, 2010).

Del año 2001 al 2007 crece el número de escuelas multigrado en relación con el resto de instituciones de I y II ciclo. Del total de estos centros educativos, en el año 2001, el 44\% eran unidocente y en el 2007 el $49.75 \%$, las cuales atendían el $8.93 \%$ del total de las niñas y los niños que asistían a la educación primaria en nuestro país, lo que representaba un aumento de un 1\% en relación al 2001; sin embargo, el porcentaje de mujeres matriculadas se mantiene durante este periodo (Chaves, García y Alvarado, 2010). 


\section{Aprobación, exclusión y repitencia de la población estudiantil del 2001 al 2007}

El porcentaje de aprobación de la población estudiantil de las escuelas multigrado del 2001 al 2007 fue de alrededor de un 87\%, superior en 4 puntos al del total de instituciones de I y II ciclo, como se evidencia en el gráfico N. ${ }^{\circ} 1$ (Chaves, García y Alvarado, 2010).

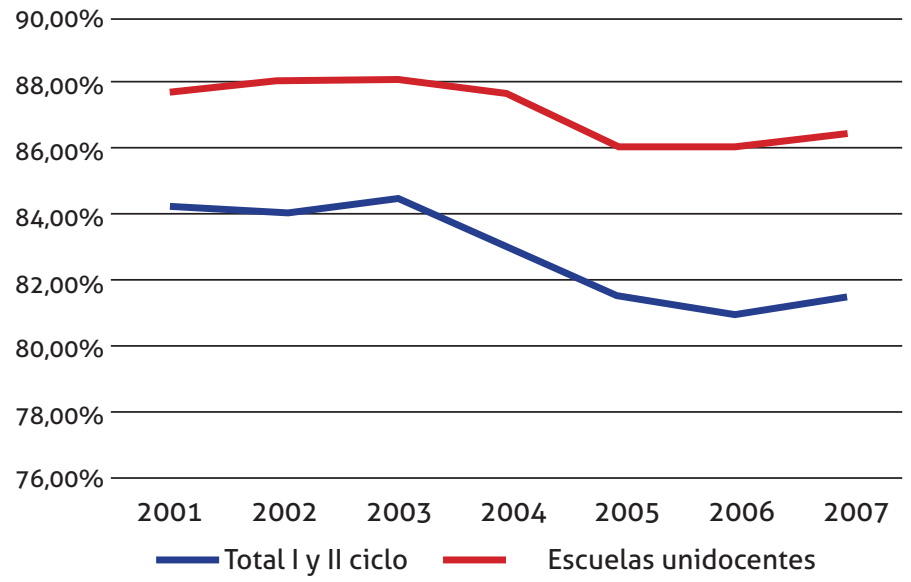

Figura 1. Aprobación total I y II ciclo y Escuelas Unidocentes

Nota: Departamento de estadística del MEP.

En relación con la reprobación durante este periodo, se mantiene en alrededor de un 7\%. Sin embargo, sí hay disminución de este porcentaje en las escuelas unidocentes en relación con el periodo 1992-2001 de acuerdo con datos del Programa Estado de la Nación (2002) que presentó un promedio de reprobación de un 11\%.

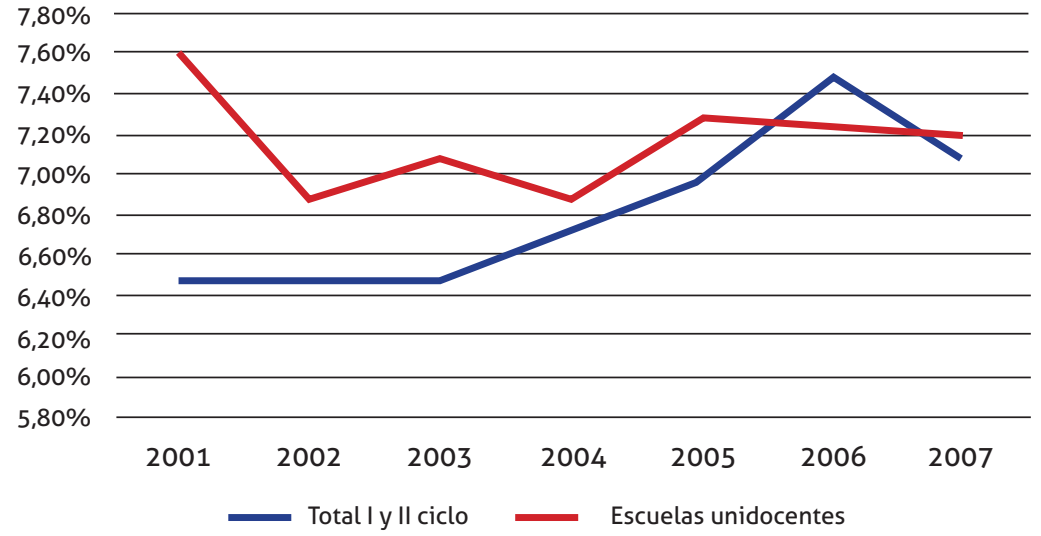

Figura 2. Reprobados Total I y II ciclo y Escuelas Unidocentes

Nota: Departamento de estadística del MEP. 
En cuanto a la exclusión en el mismo lapso de tiempo se evidencia una disminución porque pasa de un $6.2 \%$ en el 2001 a un $3.8 \%$ en el 2006, inferior a los porcentajes que presenta el conjunto de estas escuelas, como se muestra en la figura N. ${ }^{\circ} 1$

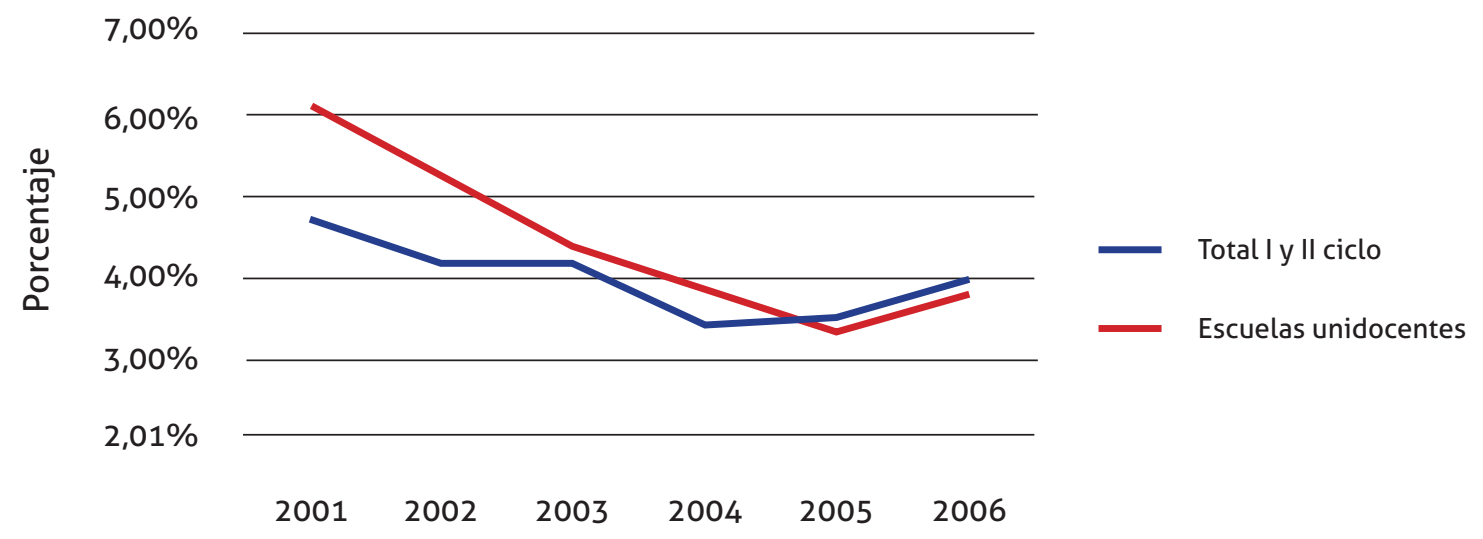

Figura 3. Exclusión del total I y II ciclo y de Escuelas unidocentes

Nota: Departamento de estadística del MEP.

Estos datos evidencian un mejoramiento del rendimiento escolar de la población estudiantil de las escuelas unidocentes en relación con la totalidad del estudiantado de I y II ciclo del país, según datos del MEP $(1997,2006)$ y del Proyecto del Estado de la Nación (2002). Este mejoramiento podría estar vinculado a la implementación del Programa Nacional de Escuelas Unidocentes del MEP, de un nuevo "Modelo Pedagógico de las Escuelas Unidocentes", a partir del año 2001, con base en la experiencia iniciada en el año 1997. Este se divulgó mediante talleres, visitas de acompañamiento para asesorar al personal técnico y administrativo de las distintas Direcciones Regionales de Educación del país, y también podría relacionarse con el aumento de nivel profesional del personal docente, como se muestra en la figura 4, del siguiente apartado (Chaves, García y Alvarado, 2010).

\section{- Características sociodemográficas del personal docente}

En relación con las características del personal docente, se obtuvo que la proporción de mujeres que laboran en estos centros educativos es ligeramente mayor (1.8 \%) a la proporción de hombres. Además, 9 de cada 10 docentes desea continuar laborando en este tipo de instituciones, una posible razón es el hecho de que 6 de cada 10 poseen el nombramiento en propiedad. El $50 \%$ de este personal tiene el grado académico de licenciatura en educación primaria y el $20 \%$ el de bachillerato en la misma especialidad. La mayoría (35\%) son graduados de la Universidad Central Costarricense (UCC), seguida por la Universidad Nacional Estatal a Distancia (UNED), de la cual se graduó el $22 \%$. Solamente el $4.5 \%$ son graduados de la Universidad Nacional, única institución de educación superior que cuenta con carrera 
de educación rural, formación que no se está aprovechando. Esta información muestra que el personal docente ha cumplido con un proceso de formación profesional, lo que le ofrece idoneidad para ejercer su función docente. Estos datos coinciden con los reportados por el Departamento de Estadística del Ministerio de Educación Pública (MEP), donde se evidencia que del 2001 al 2007 se da un incremento importante en los procesos de titulación de este personal docente (Chaves, García y Alvarado, 2010).

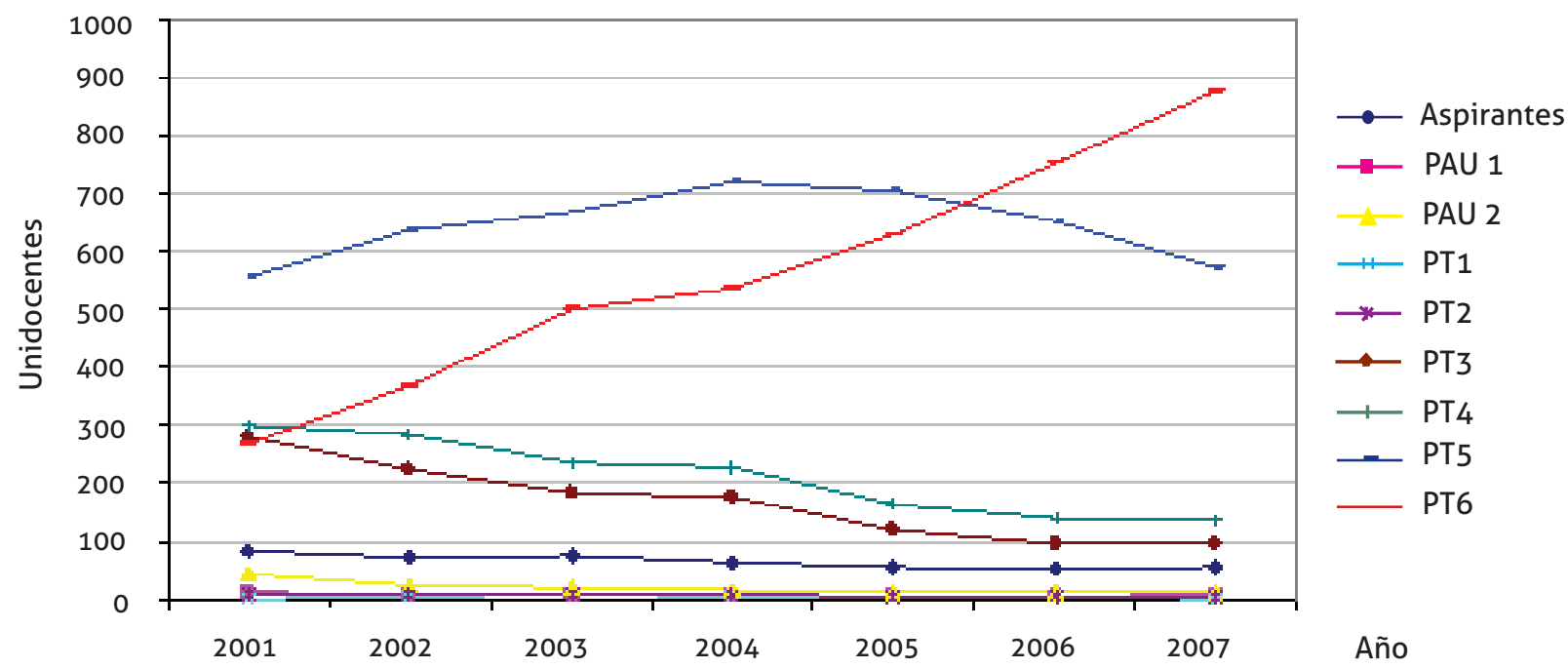

Figura 4. Distribución absoluta de los docentes por grupo profesional 2001-2007

Nota: Departamento de estadística del MEP.

\section{- Programas de estudio que recomienda el Ministerio de Educación Pública para las escuelas unidocentes a la luz de las nuevas tendencias pedagógicas.}

En el contexto de esta investigación, el MEP no contempla un programa de estudios específico para las escuelas unidocentes, por lo que estas utilizan el programa de estudio de I y II ciclo de la Educación General Básica, el cual tiene su fundamento en la política educativa hacia el Siglo XXI (Chaves, García y Alvarado, 2010).

En cuanto a este programa, el $65 \%$ de los docentes consideran que no se adecúa a las características de la población estudiantil de escuelas unidocentes. El 80 \% considera necesario que el MEP elabore un programa de estudio para escuelas unidocente, con temas y objetivos acordes a este tipo de escuelas. Esta valoración muestra el interés por un modelo curricular contextualizado (Chaves, García y Alvarado, 2010). 


\section{- Actividades de educación continua a las que asiste el personal docente de las escuelas unidocentes.}

Solamente el $10 \%$ de docentes contó con alguna capacitación por parte del Ministerio de Educación Pública (MEP) para iniciar su labor profesional en escuelas unidocentes (Chaves, García y Alvarado, 2010).

El 40 \% declaró recibir alguna asesoría para trabajar en este tipo de centros educativos por parte del MEP o alguna otra institución. 7 de cada 10 docentes recibe estas actividades de educación continua 1 o 2 veces al año. A criterio de quienes recibieron alguna asesoría, 1 de cada 3 señala que los temas tratados surgen de las necesidades de los docentes participantes (Chaves, García y Alvarado, 2010).

Los temas más comunes en las actividades de desarrollo profesional recibidas por los docentes son los que se refieren a asignaturas básicas, recibida por 6 de cada 10 docentes, y en segundo lugar las asesorías en informática educativa recibidas por 4 de cada 10 docentes. Por el contrario, las actividades de educación continua menos recibidas son las que se refieren a trabajo comunitario (4 \%). De las asesorías recibidas, asignaturas básicas e informática educativa fueron calificadas como buenas por 1 de cada 3 docentes, siendo las asesorías mejor calificadas en cuanto a calidad se refiere; por el contrario, las asesorías en inglés son las de menos calidad en opinión de 4 de cada 10 docentes. Estos datos muestran la necesidad de que el MEP, las universidades y otras instituciones ofrezcan mayores oportunidades de desarrollo profesional al personal de las escuelas unidocentes (Chaves, García y Alvarado, 2010).

\section{Características de las escuelas unidocentes: fortalezas y limitaciones}

Las características más relevantes sobre las escuelas unidocentes, en relación con sus fortalezas y limitaciones, se presentan a partir del análisis cuantitativo y cualitativo de la información recolectada. Se organizan a partir de las siguientes temáticas: gestión y organización administrativa, organización del tiempo, organización del espacio, planeamiento, práctica pedagógica, relación docente-estudiante, y relación docente, familia y comunidad (Chaves, García y Alvarado, 2010).

\section{- Gestión y organización administrativa de las escuelas unidocentes}

El personal de estas escuelas manifiesta tener un recargo de funciones tanto administrativas como docentes, debido a las características propias del quehacer cotidiano. Señalan el exceso de trabajo y la falta de tiempo para realizar todas las actividades que demanda el laborar en estos centros: dirección, docencia, conserjería, trabajo con la comunidad, trámites burocráticos, además que se les exige por parte del MEP realizar actividades tales como: recolección de dinero para diferentes instituciones del estado y organizaciones no gubernamentales, o el levantamiento de información, entre otras. Esto se une a la falta de formación en administración educativa, que únicamente cerca del $6 \%$ la posee. Al respecto, una de las maestras que se observó indica: "Yo he trabajado con Dirección 3, y como maestra de un grupo 
regular, pero nada se compara con el trabajo que demanda una escuela unidocente", y esta era una de las dos docentes que poseen el título de administración educativa. Es importante resaltar que, en el trabajo de campo, fue evidente la importancia de esta formación para el desarrollo de estos centros educativos (Chaves, García y Alvarado, 2010).

Estas instituciones educativas no cuentan con conserjes y solo en algunos casos tienen el apoyo de personal de cocina, por lo que las tareas que involucran estas actividades son asumidas por la docente, las niñas y los niños, o por algunos miembros de sus familias. En una de las escuelas visitadas, la educadora también desempeña algunas actividades en la cocina (Chaves, García y Alvarado 2010).

Estos centros educativos al contar con baja matrícula, se les asigna poco presupuesto por parte del MEP, lo que implica no contar con recursos económicos suficientes para cumplir con todas las obligaciones propias de una escuela: mantenimiento, adquisición de material didáctico, pago de servicios públicos, pago de contador, alimentación, mejoramiento de la infraestructura, entre otras. Esta situación conlleva que tanto la docente como el Patronato Escolar y la Junta de Educación se ingenien estrategias para solventar estas necesidades. Es importante señalar que también son comunidades rurales con poca población y bajos recursos económicos, por lo que los ingresos que se generan en estas actividades son aportados por las mismas familias (Chaves, García y Alvarado, 2010).

La falta de presupuesto se traduce en que, de acuerdo con el criterio del personal docente, la construcción de los centros educativos es insatisfactoria o regular en un 70 \% y los servicios sanitarios en un $75 \%$. Asimismo, más de la mitad de este personal califica la iluminación, la ventilación y el mobiliario de las aulas como insatisfactorio y regular (Chaves, García y Alvarado, 2010).

El $17 \%$ de estos centros educativos no cuentan con luz eléctrica, el 40\% no tiene agua potable, el $77 \%$ carece de teléfono y el $90 \%$ no posee conexión a internet, lo que representan condiciones adversas para ofrecer una educación relevante y pertinente a la población estudiantil de las zonas rurales (Chaves, García y Alvarado, 2010).

En contraste, es importante mencionar que tres de las escuelas unidocentes visitadas durante este estudio presentan mejores condiciones de espacio físico, cuentan con luz, agua potable y teléfono, dos cuentan con computadoras, en las otras dos son aportadas por las educadoras y ninguna tiene conexión a internet (Chaves, García y Alvarado, 2010).

En cuanto al material didáctico, el personal docente indica que este es aportado por el Programa Nacional de Escuelas Unidocentes-PRONEU- (24.5 \%), por otras instancias del MEP (17.3 \%), por las familias (14.6 \%) y la Junta Escolar (14 \%). Sin embargo, el PRONEU funcionó del 1997-2007, fue eliminado por el MEP en la administración anterior (2006-2010) y sus funciones reasignadas al Departamento de Educación Primaria. Las unidocentes que participaron en el trabajo de campo indican que ellas solicitan a las familias una cuota mensual para poder solventar los gastos en este rubro y aportan de sus ingresos personales (Chaves, García y Alvarado). 
Es evidente que es difícil realizar una gestión administrativa eficiente con todas las limitaciones que tienen las escuelas unidocentes, lo que pone en duda el cumplimiento del derecho a una educación de calidad para la niñez costarricense (Chaves, García y Alvarado, 2010).

\section{- Organización del tiempo}

La mayoría (60 \%) de las escuelas unidocentes trabaja en horario de 7:00 a.m. a 1:15 p.m. y el resto en un horario para atender a un grupo de niñas y niños de 7:00 a 11:20 a.m. y otro de 11:30 a.m. a 3:20 p.m. los cuales se alternan. Estos estudiantes se encuentran en desventaja porque reciben únicamente de 20 a 22 lecciones semanales. Es importante indicar que el MEP recomienda que todas las escuelas unidocentes trabajen el horario ampliado, pero de acuerdo con lo que menciona una de las educadoras visitadas, si la escuela tiene más de 20 estudiantes se autoriza el horario de doble jornada (Chaves, García y Alvarado, 2010).

En el horario de las escuelas unidocentes se ofrecen las siguientes materias: español, matemática, ciencias, estudios sociales y agricultura. Además, existe un periodo inicial que se denomina "círculo de la armonía", otro que se conoce como el "círculo de la creatividad" en el que se realizan actividades de arte, música y educación física debido a que en la mayoría de estas escuelas no se ofrecen las materias especiales. Asimismo, al final de la jornada existe otro momento que se denomina "Cierre pedagógico" donde se recapitula los aprendizajes obtenidos durante el día. Esta distribución de tiempo es considerada muy positiva por el personal de las escuelas unidocentes porque contribuye a "crear un ambiente propio para la asimilación de conocimientos, la creatividad, y el desarrollo personal y social" (Chaves, García y Alvarado, 2010).

En relación con las "materias especiales", los datos muestran que únicamente en el 26 $\%$ de las escuelas unidocentes se brindan y entre estas la que más se imparte es religión, seguida por educación física. En opinión de los unidocentes (95 \%) es importante que sus estudiantes reciban estas materias pues contribuyen al desarrollo integral. En el caso de inglés, únicamente se ofrece en el $31 \%$ de las escuelas unidocentes. Estos datos demuestran las desigualdades que se presentan dentro del Sistema Educativo Nacional (Chaves, García y Alvarado, 2010).

En el trabajo de campo que se realizó en cuatro instituciones unidocentes se evidenció que la jornada diaria es utilizada de manera flexible, hay libertad para que las niñas y los niños continúen trabajando durante el recreo si no han concluido. En la mayoría de las observaciones el tiempo escolar se utilizó adecuadamente, la población estudiantil se mantenía ocupada realizando alguna labor individual, grupal, en las áreas del aula o en el espacio exterior, sin embargo, en algunas ocasiones, las niñas y los niños tenían que esperar mientras la maestra revisaba asignaciones o explicaba algún contenido. Estos periodos se tornaban, en algunos casos, extensos lo que promovió la pasividad y el aburrimiento del estudiantado (Chaves, García y Alvarado, 2010). 


\section{- Organización del espacio en el aula}

Las escuelas unidocentes presentan problemas de infraestructura y mobiliario, como se mencionó anteriormente, y solo el $52 \%$ del personal docente considera satisfactorias las dimensiones del espacio físico para el número de estudiantes que se atiende. Esto se comprobó en una de las escuelas visitadas donde la educadora atendía a las niñas y los niños de primer grado en el corredor (Chaves, García y Alvarado, 2010).

En las escuelas unidocentes exitosas se observó que el personal docente acomoda los pupitres en pequeños grupos por grado, y no en filas, en cada uno de estos espacios se ubica una pizarra donde la maestra presenta la materia, da explicaciones y hace anotaciones, tal y como lo recomienda el MEP. En estas aulas se encuentra una mesa en la que se coloca en un folder el planeamiento diario para cada grado, donde las niñas y los niños líderes del nivel lo recogen y lo llevan al grupo de compañeros, lo leen y ejecutan las labores asignadas, la mayoría de las veces, estas labores se realizan mediante las guías o fichas de trabajo individuales que prepara la educadora. Para completar las guías, el estudiantado busca en las áreas o rincones de trabajo, los libros o el material necesario, realiza la labor e ilustra la ficha. En la realización de este trabajo es importante la ubicación de diferentes áreas en los salones de clase donde el estudiantado tenga acceso a los diferentes materiales, sin embargo, únicamente el 62 \% las tiene (Chaves, García y Alvarado, 2010).

Las escuelas unidocentes exitosas son limpias y ordenadas, cuentan con varias áreas de trabajo: lenguaje, ciencias, matemáticas, entre otras, las cuales están identificadas con rótulos o murales, en estas se encuentran algunos materiales que el estudiantado toma libremente para hacer sus trabajos escolares o cuando los ha concluido. En las paredes se colocan carteles con el nombre de las niñas y los niños líderes de la semana, las lecturas recomendadas para cada nivel y el tiempo que tienen para leerlas, entre otros (Chaves, García y Alvarado, 2010).

En una de estas escuelas se encuentra un área alusiva a la región donde se ubica la escuela con objetos propios de la provincia, el propósito de esta área es que el estudiantado se identifique con sus costumbres, tradiciones y se sienta orgulloso de su cultura y de sus raíces, lo que se promueve mediante el Programa de Interculturalidad del MEP. En este sentido, Peralta enfatiza en la necesidad de que el currículo escolar incorpore los valores culturales propios del estudiantado ya que esto contribuye a formar su identidad cultural y fortalecer su autoestima, lo que es de suma importancia en los niveles de educación inicial y primaria, "ya que en estas etapas se forjan las bases de la personalidad, así como un conjunto de competencias para el desarrollo humano" (1996, p. 60, citado en Chaves, García y Alvarado, 2010).

En términos generales, las escuelas exitosas organizan el espacio para que el estudiantado haga uso de él, lo que propicia la utilización de materiales y la interacción; en varias ocasiones se evidenció el apoyo que se dan entre sí las niñas y los niños de un mismo nivel, o de los niveles más avanzados a los más pequeños, lo que propicia el aprendizaje cooperativo (Chaves, García y Alvarado, 2010). 
En síntesis, el ambiente físico y afectivo del aula de las buenas escuelas promueve la autonomía y el intercambio del estudiantado, lo que contribuye, de alguna manera, a formar seres humanos participativos y críticos (Chaves, García y Alvarado, 2010).

\section{- Planeamiento}

Planear la labor educativa es una acción indispensable para desarrollar los procesos pedagógicos en las aulas de manera profesional y responsable. Más aún en las escuelas donde una sola maestra atiende a niñas y niños de los todos los niveles de la educación primaria, y en las diversas disciplinas: ciencias, español, matemática, estudios sociales, agricultura, artes, religión, entre otras (Chaves, García y Alvarado, 2010).

De acuerdo con las respuestas de los unidocentes, el $90 \%$ realiza diagnóstico de la población estudiantil, el $95 \%$ planifica su labor pedagógica tomando en cuenta los temas que propone el programa de estudio del MEP y utiliza de apoyo libros de texto (84 \%), así como libros de materias específicas (65\%), y alrededor del $95 \%$ evalúa mediante el trabajo de clase, la tareas y las pruebas escritas (Chaves, García y Alvarado, 2010).

Las escuelas unidocentes exitosas planean de acuerdo con la directriz del MEP, mediante las unidades didácticas correlacionadas y las minutas. Tres de las escuelas visitadas planifican su labor pedagógica grupalmente en los Comités Regionales de Escuelas Unidocentes. En reuniones mensuales concretan los objetivos, los contenidos, las actitudes, los valores y los aprendizajes por evaluar con base en las plantillas que ofrece el MEP. Posteriormente, cada docente define o adapta de manera individual las actividades de mediación a partir de las características y necesidades de sus estudiantes. De acuerdo con la opinión de las unidocentes a cargo de las escuelas exitosas, el planificar de manera grupal les permite compartir ideas, experiencias, materiales; analizar y discutir propuestas y sugerencias que enriquecen su práctica educativa. Además, indican que estos comités son importantes para el mejoramiento de los procesos educativos que llevan a cabo pues es un espacio donde se organizan actividades dirigidas al desarrollo de habilidades y conocimientos del estudiantado (oratoria, feria científica, redacción, entre otras); así como un sitio para introducir actividades de desarrollo profesional y de proyección comunitaria; aunque en algunos casos la actividad principal es el planeamiento didáctico (Chaves, García y Alvarado, 2010).

Estos comités no están en todas las regiones educativas del país, de acuerdo con los datos obtenidos en el año 2008 solo el $18 \%$ de los unidocentes participaban en ellos. En el año 2010, los comités son reglamentados por el MEP; sin embargo, a criterio del personal de estas escuelas, no siempre las autoridades regionales ofrecen las facilidades para su implementación o interfieren en su organización, cuando esta labor debería estar a cargo de los mismos unidocentes en coordinación con la Asesores-Supervisores y no a la inversa (Chaves, García y Alvarado, 2010).

Las unidocentes de escuelas exitosas realizan una planificación diaria de la labor pedagógica, llevan las guías de trabajo preparadas para cada estudiante, que en algunas ocasiones son cinco o seis por estudiante de acuerdo con los contenidos a estudiar, y además 
otro tipo de material necesario para el trabajo escolar. Todo este material está al alcance de los líderes por grado (Chaves, García y Alvarado, 2010).

Las maestras de estas escuelas por ejercer también las funciones de dirección en ciertas ocasiones se deben ausentar, en estos casos, una de las docentes que se visitó, elaboraba guías de trabajo para cada niña y niño, con el fin de que se las llevaran a la casa como tarea para que no se atrasaran en la labor escolar (Chaves, García y Alvarado, 2010).

Al igual que la mayoría de las unidocentes consultadas, las educadoras que se observaron evalúan los aprendizajes alcanzados por el estudiantado mediante la revisión del trabajo en la clase, las tareas y las pruebas escritas (Chaves, García y Alvarado, 2010).

\section{- Práctica pedagógica}

La mediación pedagógica de las escuelas visitadas se caracterizó por facilitar espacios de interacción que involucran a estudiantes del mismo nivel, de pares y de todo el grupo; de manera que se fortalece el trabajo colaborativo y el respeto por las diferencias en los procesos de aprendizaje. Esta forma de mediación se refleja en el uso de las fichas o guías de trabajo para cada nivel, la organización de los círculos de la armonía y de la creatividad, así como el cierre pedagógico. Esta organización permite que el estudiantado se exprese libremente: canten, declamen, bailen, dibujen, pinten, realicen manualidades, entre otras. Lo importante de estos periodos es que la población estudiantil se expresa y participa activamente y sus propuestas son aceptadas por la educadora para ser parte del proceso de aprendizaje. De esta manera se promueve el protagonismo del estudiantado (Chaves, García y Alvarado, 2010).

De acuerdo con la opinión de las niñas y de los niños, estas son de las actividades que más disfrutan, ya que al que constituirse en actividades lúdicas favorecen la autoexpresión. También se propicia conversaciones sobre diversos temas con la participación de niñas y niños de diferentes niveles, y se recurre al apoyo de los más grandes a los más pequeños. Estas actividades se favorecen por la organización del salón de clases en círculo, en algunos casos son por nivel, en otros por ciclo (I o II) y en otros con el grupo total. A ello contribuye la designación de estudiantes líderes que colaboran en el trabajo de aula con la docente y estudiantes. Esta organización promueve el diálogo, la participación, el intercambio entre el estudiantado y el aprendizaje cooperativo (Chaves, García y Alvarado, 2010).

El uso didáctico de las fichas o guías de trabajo se convierte en un mediador más del proceso de aprendizaje. Permite que las docentes puedan dedicar espacio de su tiempo para observar, comentar, revisar, hacer preguntas, responder dudas de manera individual y por nivel, lo que permite conocer las potencialidades de cada niña y niño. Promueve la autonomía de los procesos de enseñanza y aprendizaje, puesto que cada estudiante avanza a su propio ritmo. Este recurso, en algunas ocasiones, representa un reto cognoscitivo para el estudiantado, sin embargo, en otros momentos, se constituye en ejercicios de repetición, que no estimulan la indagación ni el pensamiento crítico y creativo (Chaves, García y Alvarado, 2010). 
La utilización de este recurso didáctico requiere de ambientes de aprendizaje con diversidad de material, las escuelas visitadas cuentan con libros de texto, libros de lectura, periódicos, revistas, lápices de colores, crayones, goma, tijeras, papeles de colores, entre otros, algunas veces aportados por las docentes. Sin embargo, carecen de material concreto que estimule la experimentación, para compensar este faltante las educadoras utilizan materiales del medio circundante para integrarlos en el proceso de aprendizaje. A pesar de ello prevalece la cultura del aprendizaje mediante el lápiz y el papel (Chaves, García y Alvarado, 2010).

En relación con lo anterior, cabe indicar que el $63 \%$ de estas escuelas no tiene computadora, y de las que se visitó solo dos cuentan con este equipo y por lo general es para el uso de la maestra (Chaves, García y Alvarado, 2010).

Las docentes observadas planifican sus actividades en forma correlacionada. Esta propuesta pretende integrar a todos los niveles escolares en un solo planeamiento y de forma contextualizada. Así, por ejemplo, un mismo tema se estudia en diferentes grados de complejidad dependiendo del nivel y posteriormente se realiza una puesta en común grupal; además se estudian diferentes asignaturas con ese mismo tema. A esta experiencia se integran también las artes, por eso durante la jornada escolar se intercalan actividades de canto, expresión corporal y gráfico plástica. En numerosas ocasiones se introducen los temas con una poesía, una lámina o un juego, y el cierre pedagógico por lo general se realiza mediante preguntas y diálogos sobre los temas estudiados (Chaves, García y Alvarado, 2010).

Una práctica rutinaria en la mayoría de las escuelas visitadas es un periodo de lectura, el cual se realiza de forma individual. Este contribuye a formar el hábito de lectura en el estudiantado. Sin embargo, sería enriquecedor que en este espacio se comentara y se aprovechara lo leído para realizar algunos cuestionamientos que dieran cuenta de procesos de análisis y de reflexión por parte del estudiantado. Este periodo diario se puede aprovechar con lectura de cuentos en voz alta a todo el grupo o a pequeños grupos por parte de la maestra o estudiantes (Chaves, García y Alvarado, 2010).

Aunque la mayoría de las docentes realizan una evaluación formativa corrigiendo el trabajo que efectúa cada estudiante en el aula, se evidenció que los cuadernos de las niñas y los niños, en algunas ocasiones, no son revisados por la educadora, por lo que se presentan errores de contenido. En algunos casos, se observó falta de conocimiento de parte de las niñas y los niños de contenidos básicos, de acuerdo con su grado (Chaves, García y Alvarado, 2010).

Las maestras aplican adecuaciones curriculares no significativas. En algunos de los casos se cuenta con el apoyo de maestras itinerantes de educación especial que visitan a las escuelas una vez a la semana para trabajar con niñas y niños con problemas de aprendizaje, aunque este no es un servicio generalizado el personal unidocente lo considera fundamental para atender las necesidades educativas especiales y les ayudan a fortalecer a las docentes los conocimientos en este campo. Asimismo favorece el acceso y la permanencia a la educación para esta población estudiantil, por lo que esta práctica debería universalizarse (Chaves, García y Alvarado, 2010). 
En cuanto a la valoración de los aprendizajes, como se indicó anteriormente, las maestras utilizan la evaluación diagnóstica para planear la labor pedagógica, la evaluación formativa y la sumativa, esta última mediante los trabajos que realiza el estudiantado en el aula, las tareas y los exámenes (Chaves, García y Alvarado, 2010).

En términos generales, la mediación pedagógica en estas escuelas muestra contradicciones en su puesta en práctica, porque en algunos momentos constituye un espacio idóneo para los procesos significativos, cooperativos, creativos y para la vida. Pero en otros momentos se transforma en un espacio rutinario y poco innovador de los procesos de enseñanza y aprendizaje. Es necesario tener en cuenta que la labor en estos centros educativos es compleja por lo que es recomendable contar con el apoyo de otra persona que colabore en la atención pedagógica o administrativa de la escuela (Chaves, García y Alvarado, 2010).

\section{- Relación docente-estudiante}

En las escuelas unidocentes visitadas se evidenció que las relaciones docente-estudiante se caracterizan por ser cordiales, respetuosas y afectivas. Estas formas de relación favorecen ambientes de aprendizaje democráticos, de confianza, libertad y armonía, que estimulan la participación del estudiantado (Chaves, García y Alvarado, 2010).

En general las docentes están atentas a escuchar las preguntas del estudiantado, se preocupan genuinamente por su bienestar y procuran crear un ambiente de espacio y colaboración (Chaves, García y Alvarado, 2010).

Las maestras señalan que tienen una baja deserción estudiantil y se preocupan porque sus estudiantes cuenten con el bono escolar (Chaves, García y Alvarado, 2010).

\section{- Relación docente, familia y comunidad}

El 89 \% del personal de las escuelas unidocentes considera que la participación de las familias en actividades escolares es buena y muy buena. Colaboran de diversas formas: actividades para recaudar fondos, limpieza, cocina, jardín, entre otras. Asimismo, la educadora prepara charlas sobre temas de interés que ella ofrece o especialistas a las familias y la comunidad. En las observaciones realizadas se evidenció una relación cordial y de colaboración entre docente, familia y comunidad (Chaves, García y Alvarado, 2010).

Un servicio importante que presta los EBAIS a las escuelas unidocentes es que las visitan para atender a las niñas y los niños: vacunan, desparasitan, envían exámenes de laboratorio, revisan la salud dental, entre otros (Chaves, García y Alvarado, 2010).

En los contextos donde se ubican las escuelas unidocentes, las maestras establecen vínculos con las familias y la comunidad, aspectos que se ven favorecidos por ser poblaciones pequeñas, ya que participan sus miembros de la Junta de Educación y del Patronato Escolar. De esta manera la comunidad contribuye al mantenimiento del centro educativo (Chaves, García y Alvarado, 2010). 
Es importante rescatar la labor de las buenas docentes que realizan su práctica con profesionalismo y compromiso. De ahí la necesidad de fortalecer la profesión docente creando Políticas Públicas de las que se deriven estrategias concretas en cuanto a: ingreso a la carrera docente, formación docente, desarrollo profesional, selección y reclutamiento al mercado laboral (MEP), salud ocupacional, prestigio, entre otras. Las investigaciones indican que uno de los elementos esenciales en el mejoramiento de la calidad de la educación es el recurso humano, es decir, el personal docente que guía, orienta, facilita los procesos pedagógicos que se desarrollan en las aulas y en los centros educativos (Murillo, 2006 y Day, 2006).

\section{Reflexiones finales}

En síntesis, se concluye que aunque se han realizado algunos esfuerzos, las escuelas unidocentes o multigrado no han sido prioridad para el Estado. Los datos dan cuenta de carencias en cuanto a infraestructura, material didáctico, equipo tecnológico, presupuesto, sobrecarga de trabajo para el personal docente, falta de oportunidades para el desarrollo profesional, falta de asignaturas especiales, entre otros. Esta realidad contribuye a segmentar la sociedad costarricense, a excluir grupos sociales y a reproducir desigualdades y pobreza. De ahí la importancia de transformar esta oferta educativa con el propósito de que contribuya a favorecer el desarrollo humano y social del país.

Un dato que emerge de esta investigación es el mejoramiento del rendimiento escolar de la población estudiantil de las escuelas unidocentes en relación con la totalidad del estudiantado de I y II ciclo del país del 2001 al 2007, según datos del MEP (1997, 2007) y del Proyecto del Estado de la Nación (2002). Este mejoramiento podría estar vinculado a la implementación del Programa Nacional de Escuelas Unidocentes del MEP, de un nuevo "Modelo Pedagógico de las Escuelas Unidocentes", a partir del año 2001, con base en la experiencia iniciada en el año 1997. También podría relacionarse con el aumento de nivel profesional del personal docente, el cual cambió significativamente en este mismo periodo. A pesar de lo anterior, dicho Programa se cerró en el año 2008.

Las escuelas unidocentes son una necesidad para el sistema educativo para la atención de las comunidades dispersas, lo que promueve el acceso, la equidad y el derecho a la educación, por lo que deben fortalecerse mediante un mayor apoyo del Estado para que estas escuelas contribuyan a promover el desarrollo humano y social del país.

El modelo pedagógico favorece aprendizajes significativos para el estudiantado por la organización espacio-temporal, la integración curricular, artística, el planeamiento correlacional y contextualizado. No obstante, se requiere mayor formación por parte de las universidades y actividades de actualización profesional.

El personal docente es clave para ofrecer una educación pertinente y relevante a la población estudiantil y a la sociedad, como lo confirman estudios nacionales e internacionales. 


\section{Recomendaciones que emergen del estudio}

- Es necesario tener en cuenta que la labor en estos centros educativos es compleja, por lo que es recomendable contar con el apoyo de otra persona que colabore en la atención pedagógica o administrativa de la escuela.

- Es necesario fortalecer la profesión docente mediante Políticas Públicas de las que se deriven estrategias concretas en cuanto a:

- Requisitos para ingreso a la carrera docente.

- Planes de estudio de diferentes universidades que reflejen idoneidad profesional en relación con número de créditos, horas de práctica, competencias, investigación y acercamiento a la diversidad de los contextos educativos, entre otros.

- Visión del desarrollo profesional como un proceso permanente, que comienza en la formación inicial y se continúa a través de las diferentes facetas de la vida profesional y personal.

- Selección y reclutamiento del personal docente mediante procedimientos que revelen dicha idoneidad profesional, más allá de la presentación y acreditación de un título universitario.

- Mayor protagonismo del docente en la política educativa y los procesos curriculares y del quehacer docente.

- Evaluación del desempeño profesional.

- Este estudio marca un panorama general y específico del acontecer de las escuelas unidocentes, que sirve de base para el desarrollo de nuevas investigaciones, las cuales deben realizarse. Algunos temas son: el aprendizaje colaborativo, el papel de los comités regionales, las asignaturas especiales, la efectividad de los equipos interdisciplinarios, el efecto de los cambios recientes de la estructura del MEP, el recargo de funciones administrativas y docentes, entre otros.

\section{Recomendaciones del personal de escuelas unidocentes}

- Reconocer salarialmente la dedicación exclusiva y la gestión administrativa.

- Atender las escuelas unidocentes como una modalidad educativa diferente.

- Restituir en el MEP el Programa exclusivo que vele por la atención de los procesos educativos de estas escuelas.

- Mayor apoyo presupuestario, de materiales didácticos, deportivos, así como recursos científicos y tecnológicos.

- Mayor actualización profesional especializada de parte de universidades y MEP.

- Contar con asesores especializados en las Direcciones Regionales.

- Contar con casa para el personal docente en las zonas donde se amerite.

- Mejorar la infraestructura y el mobiliario de los centros educativos.

- Mayor vinculación entre las escuelas unidocentes, mediante convivios, congresos, intercambio de experiencias, entre otros.

- Contar con personal docente para asignaturas especiales (inglés, informática educativa, música, arte, educación para el hogar, educación física, religión, agricultura). 
- Implementar un sistema de evaluación para docentes que considera aspectos como estrategias didácticas y conocimientos.

- Unificar criterios para completar los documentos oficiales.

- Valorar las implicaciones de la reestructuración administrativa del MEP.

- Designar el presupuesto del MEP por las características de las escuelas unidocentes y no por matricula, en tanto que realizan las mismas actividades o más que a las otras escuelas.

- Otorgar códigos itinerantes para la colaboración de la gestión administrativa, limpieza y cocina.

\section{Referencias bibliográficas}

Abarca, J. F., Gómez, O. y Peralta, A. (1987). Factores que afectan la labor de los unidocentes de la Región Chorotega, propuesta de una guía para el trabajo administrativo, algunas orientaciones generales sobre su labor. (Tesis de licenciatura). San José, Costa Rica: Universidad de Costa Rica.

Alvarado, R. (2007). Informe de acciones desarrolladas en el proyecto "Oportunidades digitales en escuelas unidocentes INTEL-FOD” (material policopiado). San José, Costa Rica: Ministerio de Educación Pública.

Alvarado, R. (1997). Líneas orientadoras del Programa Nacional de Escuelas Unidocentes (material policopiado). San José, Costa Rica: Ministerio de Educación Pública.

Alvarado, R. (s. f.). La Nueva Escuela Rural Unidocente de Costa Rica en Sistematización del Proyecto de Apoyo al SIMED. Un aporte con significancia cualitativa a la Educación Costarricense de I y II Ciclos. MEP. Proyecto SIMED. San José, Costa Rica: Ministerio de Educación.

Apple, M. (1995). La política del saber oficial: ¿Tiene sentido un curriculum nacional? En Volver a pensar la educación. Vol. I. Madrid, España: Morata.

Banco Mundial (2003). Análisis social de la educación rural en Costa Rica. Defensa de la niñez internacional. San José, Costa Rica: Autor.

Bernal, J. (1994). La planificación institucional: un enfoque centrado en la calidad de la educación. San José, Costa Rica: SIMED, Ministerio de Educación Pública.

Chaves, L., García, J. y Alvarado R. (2010). Las escuelas unidocentes en Costa Rica: fortalezas y limitaciones. San José, Costa Rica: Informe Final, Instituto de Investigación en Educación, Universidad de Costa Rica. 
Cruz, G. (1999). Propuesta de evaluación flexible para las escuelas unidocentes. Departamento de Evaluación del MEP (material mimeografiado) San José, Costa Rica: Ministerio de Educación Pública.

Day, C. (2006). Pasión por enseñar. La identidad personal y profesional del docente y sus valores. Madrid: Narcea.

Giroux, H. (1995). Teoría y resistencia en educación. México: Siglo XXI.

Mata, A. (2009). Entrevista personal. San José, Costa Rica: Ministerio de Educación Pública. 29 de mayo.

McLaren, P. (1997). Pedagogía crítica y cultura depredadora. Barcelona: Paidós.

Ministerio de Educación Pública. (1987). Guía para el desarrollo del curriculum en escuelas unidocentes Ministerio de Educación Pública. San José, Costa Rica: Autor.

Ministerio de Educación Pública. (1990). Programa de Estudio para el I y II ciclo de la Educación General Básica. San José, Costa Rica: Autor.

Ministerio de Educación Pública. (1994). Política educativa hacia el siglo XXI. San José: Costa Rica: Autor.

Ministerio de Educación Pública. (1997). Programa para el mejoramiento integral de la calidad de la educación de las escuelas unidocentes. San José, Costa Rica: Autor.

Ministerio de Educación Pública. (2006). Ley fundamental de educación pública. San José, Costa Rica: Autor.

Ministerio de Educación Pública. (2007). Cuadro de escuelas unidocentes y Dirección 1 con grupos heterogéneos de preescolar por región educativa en Costa Rica. San José, Costa Rica: Departamento de Estadística, Ministerio de Educación Pública.

Molina, Z. (1984). Proyecto de Reestructuración de Escuelas Unidocentes: Aplicación Experimental de un "Modelos de Escuela Unidocente sin grados en la Región de Turrialba. San José, Costa Rica: Ministerio de Educación Pública.

Murillo, J. (2006). La formación docente: una clave para la mejora educativa. En Modelos innovadores en la formación inicial docente. Una apuesta por el cambio. Santiago, Chile: Oficina Regional para América Latina y el Caribe (PRELAC). 
Programa Estado de la Nación en Desarrollo Humano Sostenible. (2002). Primer Estado de la Educación. San José, Costa Rica: CONARE.

Programa Estado de la Nación en Desarrollo Humano Sostenible. (2008).Segundo Estado de la Educación. San José, Costa Rica: Consejo Nacional de Rectores (CONARE).

Programa Regional Educativo de América Latina. (2008). Segundo Estudio Regional Comparativo y Explicativo UNESCO-ORELAC. Recuperado de HTTP://WWW.PREAL. ORG/GRUPO.3ASP?ID NOTICIA=167\&ID GRUPO=3

PREAL. (2006). "La educación de la primera infancia en la mira del informe Educación para Todos" En Boletín PREAL mayo 2006 Montevideo: Programa de Promoción de la Reforma Educativa en América Latina y el Caribe.

Sanabria, C. (1981). Problemas más relevantes que afectan la labor educativa de los unidocentes. San José, Costa Rica: Universidad de Costa Rica.

Torres, N. y Zamora, J. C. (2007). Escuelas multigrado y unidocentes en Costa Rica. Semillero de la educación rural. Heredia, Costa Rica: Centro de Investigación y Desarrollo de la Educación Rural. Dirección de Educación Rural, Proyecto PADES. Universidad Nacional.

Universidad de Costa Rica-UNICEF. (2008). VI Informe del Estado de los Derechos de la Niñez y la Adolescencia en Costa Rica. San José, Costa Rica: PRIDENA, Universidad de Costa Rica y UNICEF.

Universidad Nacional. (2008) División de Educación Rural. Recuperado de http://www.una. ac.cr/Division-/principaldivisionrural.htm 